International Journal of Pure and Applied Mathematics

Volume 94 No. 5 2014, 693-696

ISSN: 1311-8080 (printed version); ISSN: 1314-3395 (on-line version)

url: http://www.ijpam.eu

doi: http://dx.doi.org/10.12732/ijpam.v94i5.6

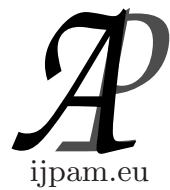

\title{
AN IMPROVED POISSON APPROXIMATION FOR BERNOULLI RANDOM SUMMANDS
}

\author{
K. Teerapabolarn \\ Department of Mathematics \\ Faculty of Science \\ Burapha University \\ Chonburi, 20131, THAILAND
}

\begin{abstract}
This paper gives a new bound for the total variation distance between the distribution of random sums of independent Bernoulli random variables and an appropriate Poisson distribution. The bound in this study is sharper than that reported in [2]. Two examples have been given to illustrate the result obtained.
\end{abstract}

AMS Subject Classification: 62E17, 60F05, 60G05

Key Words: Bernoulli random variable, Poisson approximation, random sums, total variation distance

\section{Introduction}

Let $X_{1}, X_{2}, \ldots$ be a sequence of independent Bernoulli random variables, each with probability of success $p_{i}=1-q_{i}=P\left(X_{i}=1\right)=1-P\left(X_{i}=0\right)$. If $N$ is a non-negative integer-valued random variable and independent of the $X_{i}$ 's, then $S_{N}=\sum_{i=1}^{N} X_{i}$ is the random sums of $N$ independent Bernoulli random variables. Let $\lambda_{N}=\sum_{i=1}^{N} p_{i}, \lambda=E\left(\lambda_{N}\right)$ and $U_{\lambda}$ a Poisson random variable with mean $\lambda$. For approximating the distribution of $S_{N}$ by a Poisson distribution with mean $\lambda$, Yannaros [2] gave a bound for the total variation

Received: March 26, 2014

(c) 2014 Academic Publications, Ltd. url: www.acadpubl.eu 
distance between the distributions of $S_{N}$ and $U_{\lambda}$ as follows:

$$
d_{T V}\left(S_{N}, U_{\lambda}\right) \leq E\left|\lambda_{N}-\lambda\right|+E\left(\frac{1-e^{-\lambda_{N}}}{\lambda_{N}} \sum_{i=1}^{N} p_{i}^{2}\right)
$$

where $d_{T V}\left(S_{N}, U_{\lambda}\right)=\sup _{A \subseteq \mathbb{N} \cup\{0\}}\left|P\left(S_{N} \in A\right)-P\left(U_{\lambda} \in A\right)\right|$.

In this paper, we focus on giving a new bound for the total variation distance between the two such distributions, which is in Section 2. In Section 3, some examples are given to illustrate the main result, and the conclusion of this study is presented in the last section.

\section{Result}

The following theorem presents a bound for $d_{T V}\left(S_{N}, U_{\lambda}\right)$, which is the desired result.

Theorem 2.1. Let $\theta_{N}=\frac{\sum_{i=1}^{N} p_{i}^{2}}{\lambda_{N}}$, then we have that

$$
\begin{aligned}
d_{T V}\left(S_{N}, U_{\lambda}\right) \leq & \min \left\{\min \left\{1, \sqrt{\frac{2}{e \lambda}}\right\} E\left|\lambda_{N}-\lambda\right|,\left(1-e^{-\lambda}\right) \frac{\operatorname{Var}\left(\lambda_{N}\right)}{\lambda}\right\} \\
& +\min \left\{E\left(\frac{1-e^{-\lambda_{N}}}{\lambda_{N}} \sum_{i=1}^{N} p_{i}^{2}\right), 0.46 E\left(\frac{\theta_{N}}{\left(1-\theta_{N}\right)^{3 / 2}}\right)\right\}
\end{aligned}
$$

Proof. It follows the fact that

$$
\begin{aligned}
d_{T V}\left(S_{N}, U_{\lambda}\right) & \leq d_{T V}\left(S_{N}, U_{\lambda_{N}}\right)+d_{T V}\left(U_{\lambda_{N}}, U_{\lambda}\right) \\
& \leq \sum_{n=0}^{\infty} P(N=n) d_{T V}\left(S_{n}, U_{\lambda_{n}}\right)+d_{T V}\left(U_{\lambda_{N}}, U_{\lambda}\right)
\end{aligned}
$$

The best results in [1] and [3] showed that

$$
d_{T V}\left(S_{n}, U_{\lambda_{n}}\right) \leq \frac{1-e^{-\lambda_{n}}}{\lambda_{n}} \sum_{i=1}^{n} p_{i}^{2}
$$

and

$$
d_{T V}\left(S_{n}, U_{\lambda_{n}}\right) \leq \frac{0.46 \theta_{n}}{\left(1-\theta_{n}\right)^{3 / 2}}
$$


Also by applying two results in [1], we have that

$$
d_{T V}\left(U_{\lambda_{N}}, U_{\lambda}\right) \leq \min \left\{\min \left\{1, \sqrt{\frac{2}{e \lambda}}\right\} E\left|\lambda_{N}-\lambda\right|,\left(1-e^{-\lambda}\right) \frac{\operatorname{Var}\left(\lambda_{N}\right)}{\lambda}\right\} .
$$

Taking the bounds in (2.3), (2.4) and (2.5) into (2.2), the result in (2.1) is obtained.

When $X_{i}$ 's are identically distributed, the following corollary is an immediately consequence of the Theorem 2.1

Corollary 2.1. Let $\widehat{n}=E(N)$, if $p_{1}=p_{2}=\cdots=p$, then we have the following:

$$
\begin{aligned}
d_{T V}\left(S_{N}, U_{\lambda}\right) \leq & \min \left\{\min \left\{1, \sqrt{\frac{2}{e \widehat{n} p}}\right\} E|N-\widehat{n}|,\left(1-e^{-\widehat{n} p}\right) \frac{\operatorname{Var}(N)}{\widehat{n}}\right\} p \\
& +\min \left\{E\left(1-e^{-N p}\right), \frac{0.46}{q^{3 / 2}}\right\} p .
\end{aligned}
$$

Remark. Let us consider

1. $\min \left\{\min \left\{1, \sqrt{\frac{2}{e \lambda}}\right\} E\left|\lambda_{N}-\lambda\right|,\left(1-e^{-\lambda}\right) \frac{\operatorname{Var}\left(\lambda_{N}\right)}{\lambda}\right\} \leq E\left|\lambda_{N}-\lambda\right|$ and

2. $\min \left\{E\left(\frac{1-e^{-\lambda_{N}}}{\lambda_{N}}\right), 0.46 E\left(\frac{\theta_{N}}{\left(1-\theta_{N}\right)^{3 / 2}}\right)\right\} \leq E\left(\frac{1-e^{-\lambda_{N}}}{\lambda_{N}}\right)$.

Thus, the bound (2.1) are sharper than the bound in (1.1).

\section{Examples}

This section gives two examples to illustrate the result in the case of $X_{i}$ 's are identically distributed.

Example 3.1. For $n(n \in \mathbb{N})$ is fixed, let $N$ be a positive integer-valued random variable with probability function

$$
P(N=k)= \begin{cases}\frac{1}{2} & , k=n \\ \frac{1}{2} & , k=2 n \\ 0 & , \text { otherwise. }\end{cases}
$$

Therefore $E(N)=\frac{3 n}{2}, \operatorname{Var}(N)=\frac{n^{2}}{4}$ and $E|N-E(N)|=\frac{n}{2}$. Let $p_{1}=p_{2}=$ $\cdots=p$, then we have

$$
d_{T V}\left(S_{N}, U_{\lambda}\right) \leq \min \left\{\min \left\{1, \sqrt{\frac{4}{3 e n p}}\right\} \frac{n}{2},\left(1-e^{-\frac{3 n p}{2}}\right) \frac{n}{6}\right\} p
$$




$$
+\min \left\{1-\frac{\left(e^{-n p}+e^{-2 n p}\right)}{2}, \frac{0.46}{q^{3 / 2}}\right\} p .
$$

Example 3.2. Let $N$ be a positive integer-valued random variable with probability function

$$
P(N=n)=\frac{1}{2^{n}}, n=1,2, \ldots,
$$

then we have $E(N)=2, \operatorname{Var}(N)=2$ and $E|N-E(N)|=1$. If $p_{1}=p_{2}=$ $\cdots=p$, then we obtain

$$
d_{T V}\left(S_{N}, U_{\lambda}\right) \leq \min \left\{\min \left\{1, \sqrt{\frac{1}{e p}}\right\},\left(1-e^{-2 p}\right)\right\} p+\min \left\{1, \frac{0.46}{q^{3 / 2}}\right\} p .
$$

\section{Conclusion}

In this study, a new bound for the total variation distance between the distribution of random sums of independent Bernoulli random variables and an appropriate Poisson distribution was obtained. It is also sharper than that reported in [2]. Thus, the bound obtained in this study is more appropriate for measuring the accuracy of the approximation.

\section{References}

[1] A.D. Barbour, L. Holst, S. Janson, Poisson approximation, Oxford Studies in Probability 2, Clarendon Press, Oxford, 1992.

[2] N. Yannaros, Poisson approximation for random sums of Bernoulli random variables, Statist. Probab. Lett., 11 (1991), 161-165.

[3] V. Zacharovas, H.K. Hwang, A CharlierParseval approach to Poisson approximation and its applications, Lith. Math. J., 50 (2010), 88-119. 